\title{
SURFACE DEFORMATION MONITORING IN PERMAFROST REGIONS OF TIBETAN PLATEAU BASED ON ALOS PALSAR DATA
}

\author{
L. M. Chen, G. Qiao*, P. Lu \\ College of Surveying and Geo-Informatics, Tongji University, China- (chenlemin,qiaogang,luping)@ tongji.edu.cn
}

Commission III, WG III/9

KEY WORDS: Qinghai Tibet Plateau; Permafrost; DInSAR; Deformation monitoring

\begin{abstract}
:
The permafrost region of Qinghai-Tibet Plateau is widely distributed with the freeze/thaw processes that cause surface structural damage. The differential interferometry synthetic aperture radar (DInSAR) can detect large scale surface deformation with high precision, thus can be used to monitor the freeze/thaw processes of frozen soil area. In this paper, the surface deformation pattern of Qinghai-Tibet railway was analyzed by using the PALSAR 1.0 raw data of the ALOS satellite (L band) and 90m resolution SRTM DEM data, with the help of two-pass DInSAR method in GAMMA software, and the differential interferograms and deformation maps were obtained accordingly. Besides, the influence of temperature, topography and other factors on deformation of frozen soil were also studied. The following conclusions were obtained: there is a negative correlation between deformation and temperature, and there is a delay between the deformation change and that of temperature; deformation and elevation are positively correlated; the permafrost deformation is also affected by solar radiation that could form variable amplitude variation
\end{abstract}

\section{INTROUCTION}

The permafrost region of the Qinghai-Tibet Plateau is the highest and largest permafrost area in the middle and low latitudes of the world, and its distribution is shown in Figure 1. Permafrost is below $0^{\circ} \mathrm{C}$ and contains ice in rocks and soil. Permafrost is very sensitive to the temperature of the soil medium, rich in underground ice (Zhou and Guo, 1982). The annual variation process of permafrost activity layer can be divided into four stages: summer melting process, autumn freezing process, winter cooling process and spring warming process ( $\mathrm{Li}$ and Cheng, 1996). Therefore, the surface of permafrost shows seasonal frost heave and melting phenomenon, frost heave and melting alternately will lead to the destruction of geological environment, causing collapse, landslides and debris flow and other geological disasters. Known as the "Heaven Road", the Qinghai-Tibet Railway is the longest line of plateau permafrost railway with highest elevation in the world. In order to ensure the normal operation of the Qinghai-Tibet Railway, the Qinghai-Tibet Plateau permafrost deformation monitoring work is particularly important.

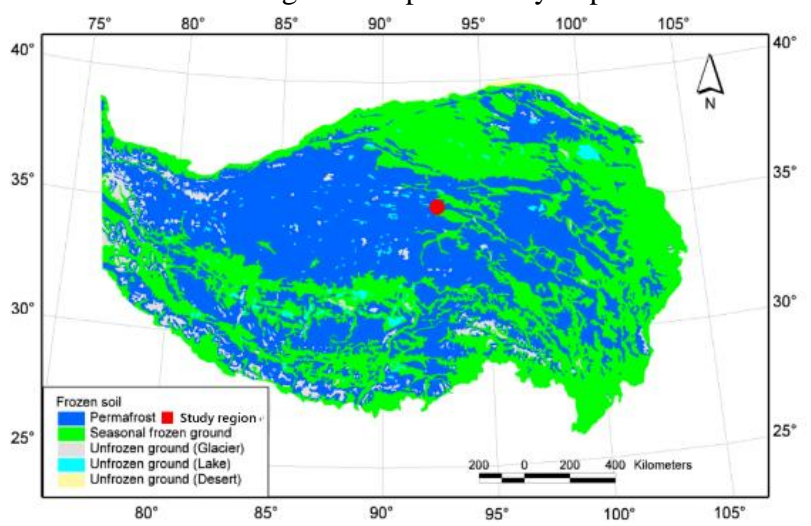

* Corresponding author: Gang Qiao
Figure 1 .Distribution pattern of permafrost in the QinghaiTibet Plateau (after Li and Cheng, 1996)

The traditional method for deformation monitoring of permafrost in plateau region is carried out by embedding settler or long-term GPS survey and leveling. Although it can obtain high-precision data, only a few limited sites can be observed due to the limited conditions, and regional deformation could hardly be collected unless a lot of labor force were consumed. Interferometry Synthetic Aperture Radar (InSAR) is a new technique for space-to-earth observation. It has the advantages of all-weather, wide monitoring range, high sensitivity, strong temporal continuity and high spatial resolution. It can make up for the shortcomings of the traditional measurement methods in the frozen area for surface monitoring.

InSAR obtains information that reflects ground undulation and surface deformation by interfering SAR images covering different phases of the same region. Qin and Perissin(2015) proposed Permanent Scatterer InSAR (PSInSAR) technology to monitor the surface deformation of the urban area of the Hong Kong Special Administrative Region by using TerraSAR-X and TanDEM-X data and obtained high-precision monitoring results.

Simon (2017) used InSAR technology to perform a wide range of deformation monitoring in the permafrost region of the Qinghai-Tibet Plateau.The Differential Interferometry Synthetic Aperture Radar (DInSAR) technique based on InSAR is to remove the terrain phase in the interference phase by performing differential interference processing on the interference image of different phases covering the same region, to obtain phase change information. Wang and Li (1999) used DInSAR technology to monitor deformation of permafrost in northern Canada, which was the first application of DInSAR technology in permafrost monitoring. Xia ,et al.(2002) used the method of combining artificial angle reflector with DInSAR 
technique to monitor the vertical deformation, and got the result of millimeter precision. Berardino, et al.(2002) proposed small Baseline Subset InSAR (SBAS InSAR) technology, using a SAR image with a smaller time and spatial baseline threshold, and improved the accuracy by the least squares method. Jia (2016) used the Small Baseline Subset InSAR (SBAS InSAR) to monitor the permafrost in the Qinghai-Tibet Plateau, and obtained the rate of permafrost change in the study area from 2007 to 2009 .

\section{STUDY REGION AND DATA}

\subsection{Study region}

The study area is located along the Qinghai-Tibet Railway and the G109 national highway in Yushu, Qinghai Province. The center of the study area is near $35^{\circ} \mathrm{N}$ and $93^{\circ} \mathrm{E}$ located in the hinterland of the Qinghai-Tibet Plateau. It is above $4000 \mathrm{~m}$ in elevation, and both sides of the railway have undulating mountains, complex terrain and less vegetation cover. It can be seen from Figure2. that this area is located in the permafrost area. Yushu is located in the hinterland of the Qinghai-Tibet Plateau, with a typical plateau alpine climate, the land freezing period from November to the following year in March. The temperature change generally appears as a quadratic function distribution, with maximum temperature in July and Minimum in January.

\subsection{Data}

PALSAR sensor in ALOS satellite is an L-band active microwave sensor, the wavelength of which is about $22 \mathrm{~cm}$ with strong penetration overcoming the impact of clouds. Data used in this study is the Level 1.0 PALSAR product obtained in 2007 and 2008, with polarization mode of $\mathrm{HH}$ and two modes of FBD and FBS. The dimension of the study data is $70 \mathrm{~km} \times 70$ $\mathrm{km}$ and the viewing angle is $33.4^{\circ}$, with time span of about one year for the five SAR images: March 4, 2007, July 20, 2007, September 4, 2007, October 20, 2007 and March 6, 2008.

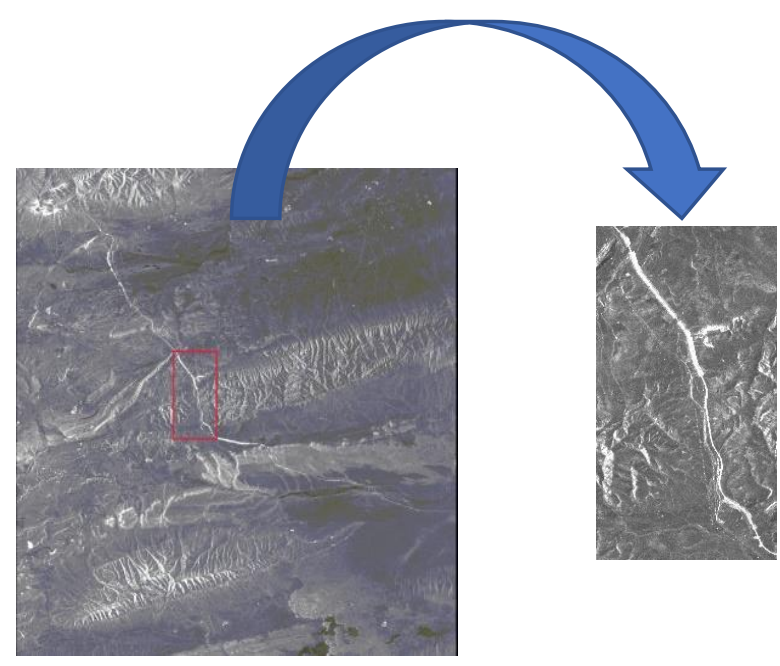

Figure 2 . Study area

In addition, this research also employs the external DEM: the V4.1 version of SRTM3 data with a resolution of $90 \mathrm{~m}$ and coverage of $92^{\circ} \sim 94^{\circ} \mathrm{E}, 34^{\circ} \sim 36^{\circ} \mathrm{N}$.

\section{DATA PPROGRESSING AND METHODOLOGY}

In this study, the first SAR image is used as the reference image, and the remaining four images as slave image. The differential interference processing is carried out for the four interference image pairs. The baseline information of the four interferometric pairs is shown in the Table 1.

\begin{tabular}{|c|c|c|}
\hline image pairs & $\begin{array}{c}\text { Space Baseline ( } \\
\mathrm{m} \text { ) }\end{array}$ & Time base ( day ) \\
\hline $20070304-20070720$ & 789.7 & 138 \\
\hline $20070304-20070904$ & 1153.1 & 184 \\
\hline $20070304-20071020$ & 1348.7 & 230 \\
\hline $20070304-20080306$ & 2679.2 & 368 \\
\hline
\end{tabular}

Table 1. Baseline information on the pair

The two-pass method uses the external acquired DEM data to simulate the terrain phase in the SAR image, generates the simulation pattern, and then combines the interferogram for differential interference, so as to obtain high-precision surface deformation information. The interference phase consists mainly of the following five parts:

Where

$$
\begin{aligned}
& \varphi=\text { the interferogram phase } \\
& \varphi_{\text {flat }}=\text { the flat phase } \\
& \varphi_{\text {topo }}=\text { the topographic phase } \\
& \varphi_{\text {noise }}=\text { the noise phase } \\
& \varphi_{\text {atmo }}=\text { the atmospheric phase } \\
& \varphi_{\text {disp }}=\text { the deformation phase } \\
& \qquad \varphi_{\text {disp }}=-\frac{4 \pi}{\lambda} \Delta r
\end{aligned}
$$

Where $\lambda=$ wave length

$$
\Delta \mathrm{r}=\text { distance difference }
$$

In addition, the expression of the flat phase is shown in equation (3).

$$
\varphi_{\text {flat }}=-\frac{4 \pi \times B \cos (\theta-\alpha)}{\lambda R_{1} \tan \theta} \Delta r
$$

When the two interferograms are obtained, the terrain phase is eliminated, here we ignore the influence of noise and atmospheric delay, and the difference phase is

$$
\Delta \mathrm{r}=-\frac{\lambda}{4 \pi} \varphi-B \sin (\theta-\alpha)-\frac{B \cos (\theta-\alpha)}{R \sin \theta} h
$$

The two-pass differential interference method is carried out according to the above principle, and the terrain phase, the flat phase and the noise phase and the atmospheric phase are sequentially removed to obtain the information of the displacement phase. The specific processing workflow is shown in Figure 3. 


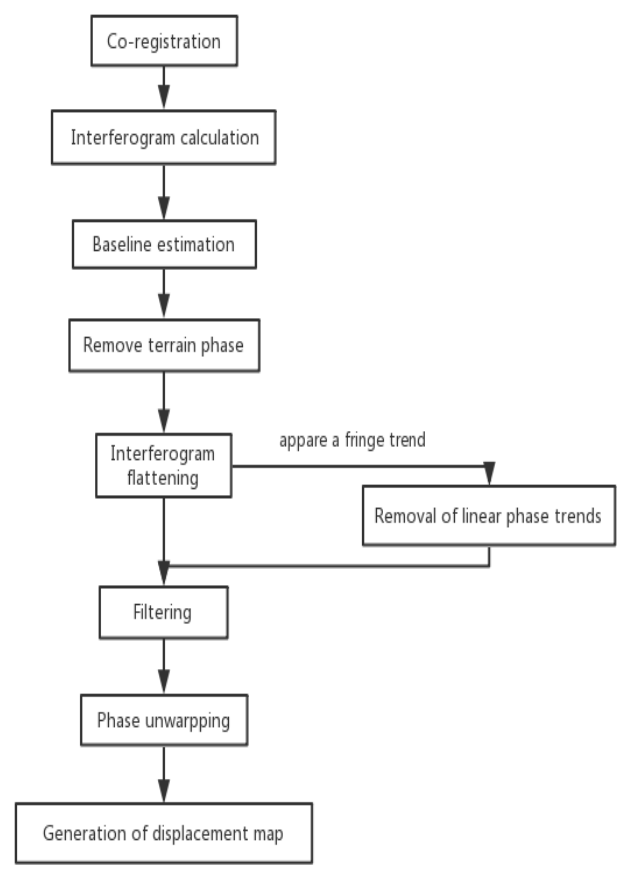

Figure 3. Two-pass method DInSAR processing flow chart

Since the three-pass difference method is easy to introduce the phase unwrapping error when the terrain phase is removed, this paper uses a $90 \mathrm{~m}$ resolution DEM data, the two-pass differential interference. SAR image obtained on March 4, 2007 is used as the reference image to make four interference image pairs with the other four images. Then image registration was performed for the four pairs with an acceptable registration accuracy. The two single look complex (SLC) images of each image pair are conjugated and multiplied to obtain the interferogram. The terrain phase is calculated using the DEM data of SRTM3 and is removed from the interferogram. The deformation phase is obtained by flattening, filtering and so on. Finally, the deformation phase in the study area is obtained and shown in Figure 4.
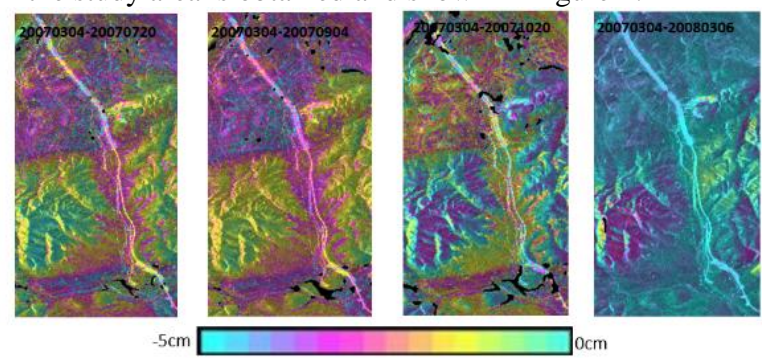

Figure 4. Deformation map of the study area

It can be seen in Figure 4 that the surface of the study area settled on July 4, 2007. The subsidence trend of the surface was further deepened on September 4, 2007. On October 20, 2007, the subsidence was significantly reduced. On March 6, 2008 deformation was significantly reduced.

\section{RESULT AND DISSCUSSION}

In order to speculate the deformation trend of the surface concretely and observe the deformation law more obviously, this paper selected nine deformation points from the study area. Among them, points $1 \sim 5$ are selected along the railway, $6 \sim 9$ is in the rough terrain area. The distribution position is shown as in Figure 5.

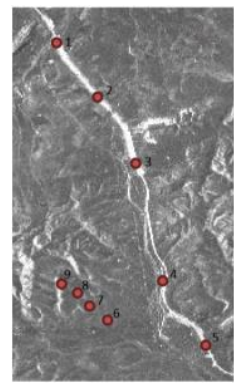

Figure 5. Distribution of deformation points selected in the study region

The elevation information and deformation information of each extracted deformation point corresponding to the different time is shown in the Table 2 .

\begin{tabular}{|l|l|l|l|l|l|}
\hline \multirow{2}{*}{ Number } & \multirow{2}{*}{$\begin{array}{c}\text { elevation } \\
(\mathrm{m})\end{array}$} & \multicolumn{4}{|c|}{ Displacement (cm) } \\
\cline { 3 - 6 } & & 0720 & 0907 & 2007 & 2008 \\
& & -2.2 & -2.6 & -1.8 & 0.7 \\
\hline 1 & 4699 & -1.7 & -2.4 & -2 & 0.9 \\
\hline 2 & 4719 & -1.4 & -1.9 & -1.5 & 0.3 \\
\hline 3 & 4714 & -1.3 & -1.9 & -1.6 & 0.3 \\
\hline 4 & 4709 & -1.3 & -2.5 & -2 & 0.8 \\
\hline 5 & 4716 & -2.6 & -2.9 & -1.5 & -0.4 \\
\hline 6 & 4604 & -2.1 & -2.4 & -1.6 & 0.2 \\
\hline 7 & 4707 & -1.5 & -2.1 & -1.3 & 0.7 \\
\hline 8 & 4738 & -0.7 & -1.3 & -0.5 & 1.3 \\
\hline 9 & 4759 &
\end{tabular}

Table 2. Deformation information of the nine test points

\subsection{The effect of temperature changes}

The monthly average temperature of the Yushu station in Qinghai from March 2007 to March 2008 was downloaded from the Chinese Meteorological Network (reference??). The trend of the monthly mean temperature and the nine deformation points is shown in Figure 6. According to this figure, the temperature gradually began to climb from zero in March 2007, reaching the highest temperature in July and August, and then gradually decreased, to $0^{\circ} \mathrm{C}$ at the end of October. In the following year in February the temperature began to gradually rise again.

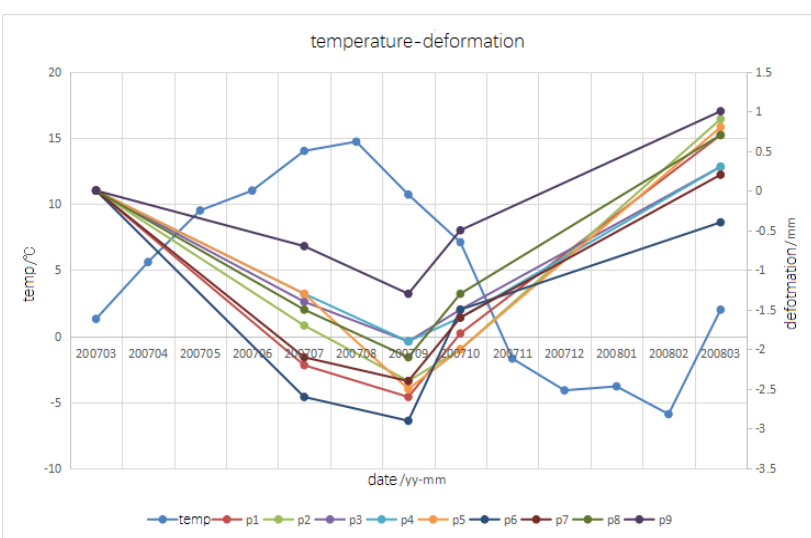

Figure 6. Temperature-Deformation Change Chart 
According to Figure 6, it can be deduced that the deformation of the deformation point is negatively correlated with the temperature change, and the variation period is about one year. From March to September, when the temperature is high, the ice in the permafrost reaches the melting point, and the ground subsidence causes deformation. Since October, the temperature began to decline, when water re-iced, the ground appeared uplift.

\subsection{Impact of terrain changes}

In addition, terrain is also an important factor to effect the surface deformation because elevation and solar radiation changes can cause the deformation to change to some extent in the law.

The deformation points $6 \sim 9$ are located in the rough terrain area from the foot of the mountain to the top of the mountain. We can observe the relationship between elevation and deformation by the elevation-deformation chart shown in Figure 7. The deformation is positively correlated with the elevation at different times. As the elevation increases, the subsidence (negative) decreases gradually, and even the surface uplift occurs

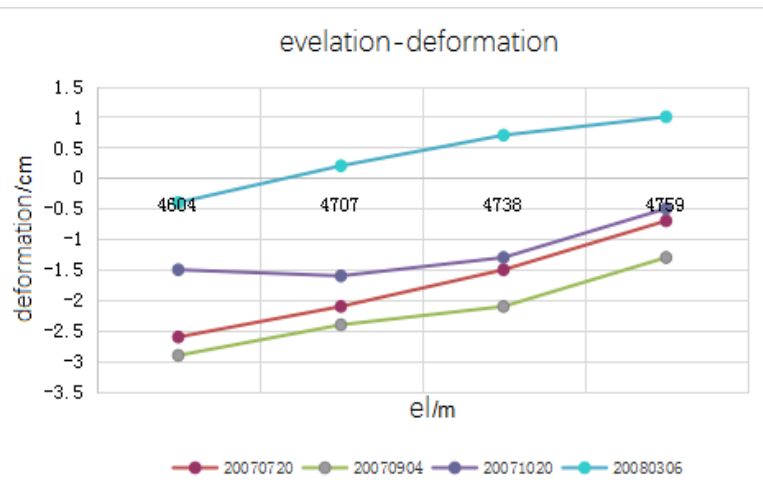

Figure 7. Elevation-Deformation Chart for test points 6-9 in the mountainous region

It can be found that there is a significant difference between the shady and sunny slope of the same mountain. In the case of the mountain area intercepted by Figure 8 , it can be found that at the same time, the deformation of the sunny slope is greater than the deformation on the shady slope; comparing the four deformation figures together, we find the range of sunny slope changing deformation is larger than the shady area.

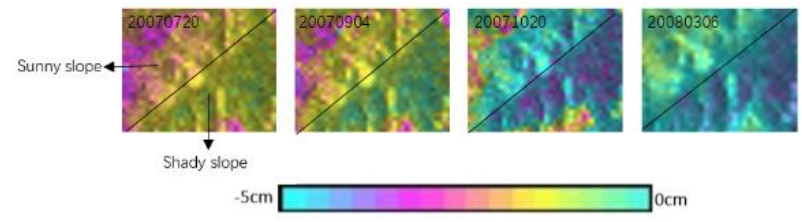

Figure 8 . The shady and sunny slope in the study region

\section{CONCLUSIONS}

According to the results obtained from this study, the general law and trend of surface deformation in permafrost area can be inferred, and we also analyzed the influence of temperature and topography. By comparing the deformation points' air temperature and deformation changes at different time, we found the deformation is negatively correlated with the temperature. By comparing the deformation points' deformation and elevation, it can be deduced that the elevation is roughly positively related to the deformation. In addition, it was found that the range of deformation at the sunny slope is larger than that at the shady slope, and at the same time, the deformation of the sunny slope is also larger than the deformation on the shady slope.

In this paper, there are still some shortcomings and problems which can be improved: the data of this study only use five images of ALOS PALSAR SAR, the differential interference results can only observe the general trend of permafrost deformation, thus cannot be a good embodiment of the law of permafrost in the continuity of time. Although some errors were removed by GAMMA software, there still are some residuals. This paper cannot obtain the surface deformation data with the field test, and can only compare the experimental results with the previous permafrost research results, which cannot be accurate analysis of the study results. In the future, we will further make up for deficiencies, improve the process, to get more accurate results.

\section{ACKNOWLEDGEMENTS}

This research was supported by the National Key Research and Development Program of China (2017YFA0603102), the National Science Foundation of China (91547210, 41771471, 41201425), and the Fundamental Research Funds for the Central Universities.

\section{REFERENCES}

Berardino P.,Fornaro G., et al. 2002.A new algorithm for surface deformation monitoring based on small baseline differential SAR interferograms.IEEE Transactions on Geoscience and Remote Sensing.40 (11),pp.2375-2383.

Daout S, Doin M, Peltzer G, et al. 2017. Large-scale InSAR monitoring of permafrost freeze-thaw cycles on the Tibetan Plateau. GEOPHYSICAL RESERCH LETTERS.44(2), pp.901-909.

Yuxiao Qin,Daniele Perissin. 2015. Monitoring Ground Subsidence in Hong Kong via Spaceborne Radar: Experiments and Validation.Remote Sensing.7(8),pp.10715-10736.

Shude Li, Guodong Cheng, 1996.Qinghai - Tibet Plateau permafrost. Gansu Culture. Lanzhou.

Wang Z, Li S, J., 1999.Detection of Winter Frost Heaving of the Active Layer of Arctic Permafrost Using SAR Differential Interograms. IEEE International Geoscience and Remote Sensing Symposium. 4, pp.1946-1948.

Xia Y, Kaufmann H, Guo X F.2002.Differential SAR interferometry using corner reflectors. IDARSS,.02,pp.12431246.

Youwu Zhou, Dongxin Guo, J., 1982.The Main Characteristics of Permafrost in China .Glacier permafrost. 4(1), pp. 1-19.

Yuanyuan Jia, et al, J., 2016.Characterization of Active Layer Thickening Rate over the Northern Qinghai-Tibetan Plateau Permafrost Region Using ALOS Interferometric Synthetic Aperture Radar Data 2007-2009.Remote Sensing. 9(1). 